\title{
Scattering in the Ultrastrong Regime: Nonlinear Optics with One Photon
}

\author{
E. Sanchez-Burillo, ${ }^{1}$ D. Zueco, ${ }^{1,2}$ J. J. Garcia-Ripoll, ${ }^{3}$ and L. Martin-Moreno ${ }^{1, *}$ \\ ${ }^{1}$ Instituto de Ciencia de Materiales de Aragon and Departamento de Fisica de la Materia Condensada, \\ CSIC-Universidad de Zaragoza, E-50012 Zaragoza, Spain \\ ${ }^{2}$ Fundacion ARAID, Paseo Maria Agustin 36, E-50004 Zaragoza, Spain \\ ${ }^{3}$ Instituto de Fisica Fundamental, IFF-CSIC, Calle Serrano 113b, E-28006 Madrid, Spain
}

(Received 1 July 2014; published 31 December 2014)

\begin{abstract}
The scattering of a flying photon by a two-level system ultrastrongly coupled to a one-dimensional photonic waveguide is studied numerically. The photonic medium is modeled as an array of coupled cavities and the whole system is analyzed beyond the rotating wave approximation using matrix product states. It is found that the scattering is strongly influenced by the single- and multiphoton dressed bound states present in the system. In the ultrastrong coupling regime a new channel for inelastic scattering appears, where an incident photon deposits energy into the qubit, exciting a photon-bound state, and escaping with a lower frequency. This single-photon nonlinear frequency conversion process can reach up to $50 \%$ efficiency. Other remarkable features in the scattering induced by counterrotating terms are a blueshift of the reflection resonance and a Fano resonance due to long-lived excited states.
\end{abstract}

Introduction.-As light-matter interaction controls an immense variety of physical processes, its modification usually leads to new phenomena. One strategy to increase this interaction is to confine the electromagnetic field in waveguides and make it interact with few level systems. It is possible nowadays to reach in this way the situation where the coherent light-matter coupling predominates over decoherence processes (the so-called strong-coupling regime), and to generate, manipulate, and store a single (or a few) photon. The ability of performing tasks with just one photon has already been demonstrated [1,2], opening the path for proposals such as optical transistors [3-5], singlephoton routers [6], one-photon lasers [7], qubit-mediated entanglement [8], or efficient photodetectors [9].

All these results have been analyzed within the rotatingwave approximation (RWA) for the photon-dipole interaction [10]. The RWA only considers the processes where light and matter exchange excitations, which is valid when the couplings are much smaller than the typical photon and qubit energies. For sufficiently strong couplings, processes involving spontaneous creation and annihilation of pairs of excitations are relevant and the RWA picture breaks down [11]. This regime of ultrastrong coupling opens the door to new physics [12,13], which is within reach for many different experimental implementations [14].

From the theoretical viewpoint, within the RWA the scattering of multiphoton wave packets by qubits is a complex problem [15-20], but the one-photon scattering is trivial. Beyond the RWA, computing the scattering of even one flying photon is difficult as subspaces with different photon numbers mix in the dynamics. This converts the problem into a many-body one for which only partial solutions exist for models that consider linear (unbounded) dispersion relations and, typically, either in the perturbative regime $(g / \omega<0.2)$ or in the localization phase $(g / \omega>1)$.

In this Letter we analyze the scattering by one flying waveguide photon by one qubit for an ample range of photon-qubit interactions that comprise the strong and ultrastrong coupling regimes, and take into account the effect of nonlinearity in the photon dispersion relation. For that, we use the framework of matrix product states [21-23] to compute the many-body dynamics. For sufficiently small couplings, we recover the RWA results where the qubit acts as a perfect mirror in resonance. However, for stronger couplings, a richer phenomenology is found: renormalization of the resonant frequency, appearance of an asymmetric Fano resonance, and existence of inelastic Raman processes.

Model and methods.-The photonic medium is represented as a chain of $L$ discrete bosonic sites (which can be considered either as bona fide coupled cavities or as a discretization of a continuous waveguide) coupled to a qubit living at site $j_{0}=0$ (see Fig. 1). The Hamiltonian of the combined system is $(\hbar=1)$

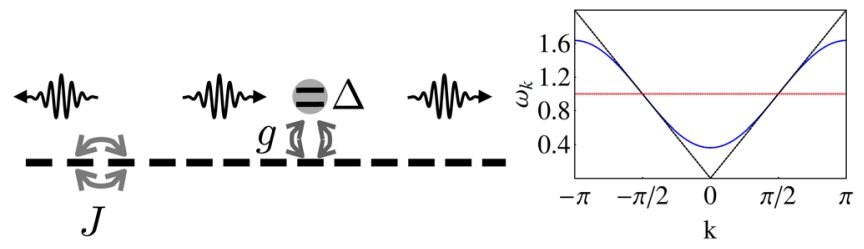

FIG. 1 (color online). Left: schematics for the considered system. Right: dispersion relation of the free photon band considered in this problem. The discontinuous line represents a linear dispersion with the same velocity at the qubit frequency $\Delta=1$. 


$$
H=\sum_{j}\left[a_{j}^{\dagger} a_{j}+J\left(a_{j+1}^{\dagger} a_{j}+\text { H.c. }\right)\right]+\Delta \sigma^{+} \sigma^{-}+g \sigma_{x} X_{j_{0}},
$$

where the first two terms represent the photons in the waveguide, the third one describes the qubit, and the fourth term is the interaction between a dipole transition and the local electric field (characterized by a strength $g$ ). In Eq. (1), $a_{j}^{\dagger}$ and $a_{j}$ create and annihilate, respectively, a photon at position $j, X_{j_{0}} \equiv a_{j_{0}}^{\dagger}+a_{j_{0}}$, and $\sigma_{x}$ and $\sigma^{ \pm}$are Pauli and ladder matrices acting onto the qubit, which has an excitation energy $\Delta$. The free-photon dispersion relation depends on both the on-site photon energy (which is taken as the frequency unit) and the hopping parameter $J$ : $\omega_{k}=1+2 J \cos (k)$. Throughout the paper we take $J=-1 / \pi$ and $\Delta=1$, so that the qubit resonance sits where the photon band is linear. Importantly, the finite bandwidth of the dispersion relation implies the existence of bound states localized in the vicinity of the qubit [24]. As shown below, these states are essential in some scattering properties, so continuum models with unbounded photon dispersion relations may present different physics. Notice though that realistic waveguides always have at least lowfrequency cutoffs.

The interaction Hamiltonian can be expressed as the sum of "rotating wave" and "counterrotating" contributions, $H_{\mathrm{int}}^{\mathrm{RW}}=g\left(\sigma^{+} a_{j_{0}}+\sigma^{-} a_{j_{0}}^{\dagger}\right)$ and $H_{\mathrm{int}}^{\mathrm{CR}}=g\left(\sigma^{+} a_{j_{0}}^{\dagger}+\sigma^{-} a_{j_{0}}\right)$, respectively. For $g \ll \Delta, H_{\text {int }}^{\mathrm{CR}}$ can be safely neglected, which greatly simplifies the calculations as $H_{\text {int }}^{\mathrm{RW}}$ conserves the total number of excitations, $N_{\text {exc }}$. For large enough couplings (as a rule of thumb when $g \geq 0.1 \Delta$ ) $H_{\text {int }}^{\mathrm{CR}}$ cannot be neglected and subspaces with different number of excitations are visited during the dynamics. Nevertheless, the full Hamiltonian still has parity $\Pi=(-1)^{N_{\text {exc }}}$ as a conserved quantity.

As mentioned, the presence of counterrotating terms converts the scattering of even a single photon into a manybody problem. Hence a brute-force computation of the time evolution is prohibitive, even for small chain lengths. Our calculations use the representation of matrix product states (MPS) to describe the wave function $[25,26]$. Whenever a many-body state is slightly entangled, as typically occurs for 1D systems in the low-energy sector [27], MPS is optimal. The complexity is not exponential anymore, as it happens for a random state, but it is polynomial with the size of the system. This allows the study of the low-energy physics by means of classical computation. Further details on both the method, its convergence, and the tests performed can be found in the Supplemental Material [28].

The simulation of the scattering process follows the following steps: (i) computation of the ground state (GS), (ii) generation of the input state comprising the GS plus one incoming photon, (iii) time evolution of the wave function, and (iv) analysis of the final wave function.
Ground and excited states.-We compute the GS by imaginary time evolution of a seed state. Within the RWA the GS is the vacuum ( 0 photons and 0 qubit excitations). However, when counterrotating terms are relevant, the GS is a nontrivial "dressed qubit," with a photon cloud bound to the two-level system. Excited bound states can also be computed by the same method, by proper orthogonalization with lower lying states. Figure 2 shows the energy of the ground state and the first bound excited states, as a function of $g$. Their spatial profile of number of photons in the cloud is rendered in the inset of Fig. 2.

For small $g$, i.e., within the RWA, the index $n$ in $E_{n}$ labels the number of excitations in the state (with $G S \equiv E_{0}$ ). The single-photon bound state $E_{1}$, already predicted in RWA models [24], does not play a role in the scattering process, as it lies outside the one-photon band. On the contrary, the $E_{3}$ energy lies inside that band, with which it hybridizes. Thus, strictly speaking, $E_{3}$ is a leaky bound mode. This complicates the computation of $E_{3}$ using MPS; Fig. 2 shows its estimated energy, obtained via numerical diagonalization of (1) for a lattice with $L=7$ sites.

Scattering simulation.-As the input state, we create on the GS a one-photon Gaussian wave packet, centered at $x_{0}$ with spacial width $\sigma$, moving towards the qubit with average momentum $k_{\text {in }}$ (and corresponding frequency $\omega_{\text {in }}$ ),

$$
|\Psi(0)\rangle=a_{\phi}^{\dagger}|G S\rangle \equiv \sum_{x} \phi_{x} a_{x}^{\dagger}|G S\rangle
$$

with $\phi_{x} \propto \exp \left[-\left(x-x_{0}\right)^{2} / 2 \sigma^{2}+i k_{\text {in }}\right]$. The time evolution of this wave gives us $|\Psi(t)\rangle$.

Useful quantities to characterize the scattering are the average local number of photons $\left\langle n_{x}(t)\right\rangle=$ $\left\langle\Psi(t) a_{x}^{\dagger} a_{x} \mid \Psi(t)\right\rangle$, its equivalent in Fourier space $\left\langle n_{k}(t)\right\rangle$, and the one photon dynamics over the GS $\phi_{x}(t)=\left\langle G S\left|a_{x}\right| \Psi(t)\right\rangle$. From the Fourier transform of the latter we can extract the transmission amplitude as $t_{k}=\phi_{k}\left(t_{f}\right) / \phi_{k}^{\text {free }}\left(t_{f}\right)$, where $t_{f}$ is a time long enough so

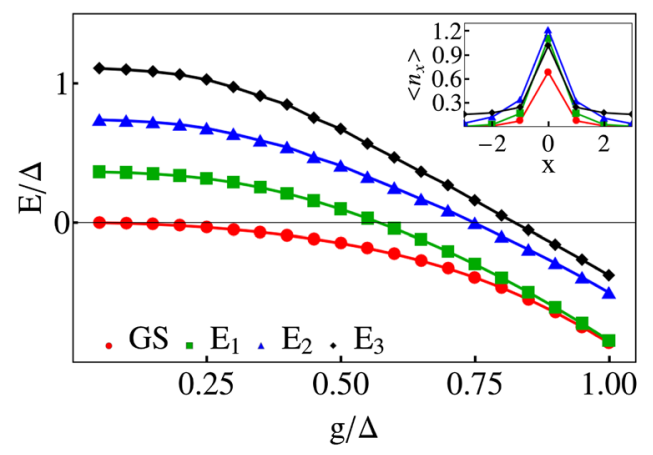

FIG. 2 (color online). Energies of bound states. Dependence with coupling $g$ of ground state and bound excited state energies. $G S$ and $E_{2}$ have even parity, while $E_{1}$ and $E_{3}$ have odd parity. The inset shows the spacial profile of the number of excitations in each bound state, for $g=0.8$. 
that the scattering process has concluded, and $\phi_{k}^{\text {free }}$ is the propagation when the dressed qubit and the incoming photon do not interact. These quantities (together with the qubit population, which we analyze in the Supplemental Material) suffice for analyzing scattering amplitudes as, in all considered cases, the computed amplitude for a generation of more than one propagating photon is negligible.

Figure 3 shows both $\left\langle n_{x}(t)\right\rangle$ and $\left\langle n_{k}(t)\right\rangle$ for two representative cases, corresponding to different $k_{\text {in }}$, and $g=0.7$. For this value of $g$, at which the RWA is not valid, the GS comprises a photon cloud around the qubit, as seen in both $\left\langle n_{x}(t=0)\right\rangle$ (at $x \approx 0$ ) and $\left\langle n_{k}(t=0)\right\rangle$ (which presents a finite value around $k=0)$. As time evolves, we observe the typical scattering evolution. After a time span of free propagation $(t \lesssim 100)$, an interaction period starts where both reflected and transmitted photon beams develop. Finally, at larger times $(t \gtrsim 300)$, the scattered photon propagates freely.

There are always reflected and transmitted elastic beams, which propagate at the same speed as the incident one. Remarkably, as shown in the Figs. 3(b) and 3(d), for some parameters there are also inelastic (Raman) processes where both reflected and transmitted wave packets propagate with a different speed to the incident one (and thus a different frequency). Notice also that, in this case, after the scattering event the photon cloud around the qubit has
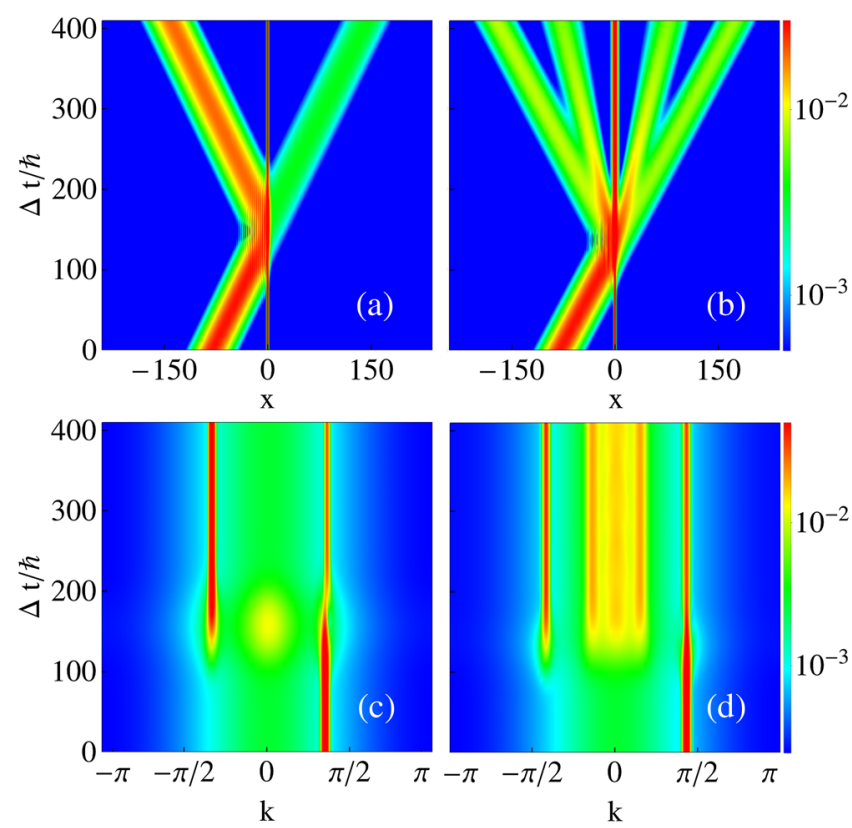

FIG. 3 (color online). Time evolution. Evolution of $\left\langle n_{x}\right\rangle$ (upper panels) and $\left\langle n_{k}\right\rangle$ (lower panels) for $\omega_{\text {in }}=0.70$ (left panels) and $\omega_{\text {in }}=0.85$ (right panels). In both cases, at $t=0$ an initial wave packet is set centered at $x_{0}=-80$ and the coupling is $g=0.7$. For $\omega_{\text {in }}=0.70$ the scattering is elastic, while for $\omega_{\text {in }}=0.85$ there is an inelastic scattering channeling too. changed, being now broader in real space (thus narrowing momentum space).

Elastic scattering.-Figure 4 renders the transmission into the elastic channel, as a function of both $\omega_{\text {in }}$ and $g$. The top panel is obtained within the RWA, while the lower panel is computed using the full Hamiltonian.

For sufficiently small $g(g \lesssim 0.3)$, the elastic transmission spectra is, both within the RWA and for the full model, characterized by a deep transmission minimum, with a spectral width that increases with $g$. The main difference is that, while within the RWA the minimum always occurs at $\omega_{\min }=\Delta$, in the full model the transmission minimum blueshifts with $g$. This shift is reminiscent of the frequency renormalization in the spin-boson model [31,32], which is a continuum model without band edges. However, the renormalization group flow predicts a redshift of the effective frequency of the qubit. Here, the waveguide presents a natural cutoff at high $\omega$, which prevents a direct application of the renormalization group. Nevertheless, in this intermediate regime the counterrotating terms can be taken into account perturbatively (see Supplemental Material), leading to an analytical condition for the spectral position of the transmission minimum, which is rendered in Fig. 4(b) (white line).

For larger $g, \quad(g>0.3)$, an asymmetric Fano-like resonance develops in the elastic transmission spectra. This feature combines a deep minimum and a strong
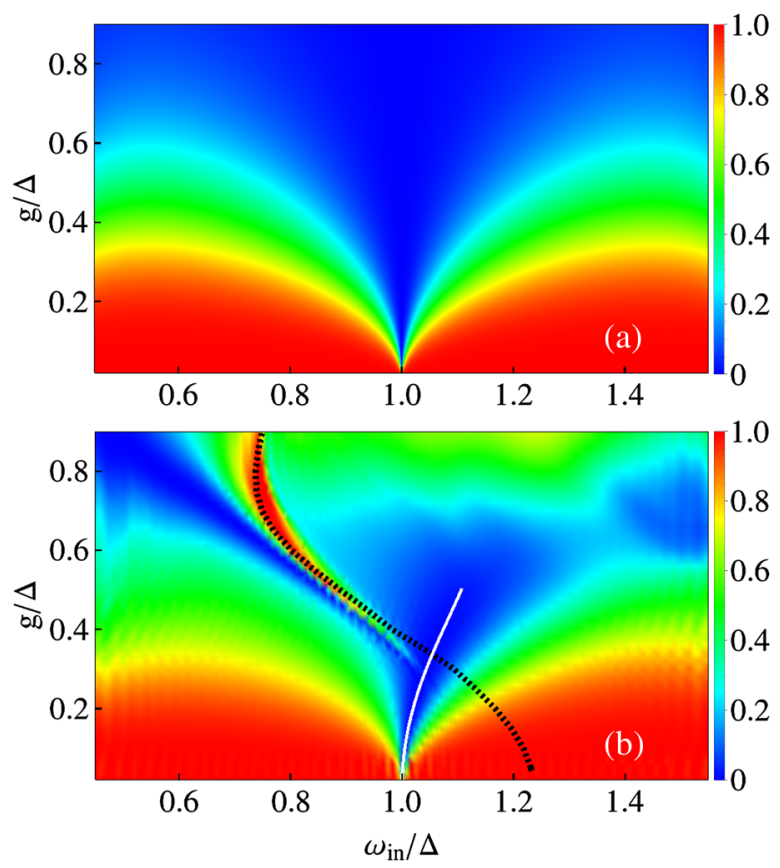

FIG. 4 (color online). Transmission as a function of both incident photon frequency $\omega_{\text {in }}$ and $g$. (a) Transmittance within the RWA. (b) Elastic transmittance in the full model. The black line marks the estimated frequency for the Fano resonance while the white line gives the estimated spectral position for the transmittance minimum (see text). 
transmission maximum, with a line width that increases monotonically with $g$. Fano resonances are the hallmark of long-lived states entering the scattering dynamics. In this case, its origin can be traced back to the leaky bound state $E_{3}$, as shown by the agreement between the frequency at which the resonance occurs and the computed energy difference $E_{3}-E_{G S}$ (black line in Fig. 2). As commented, within the RWA, the state $E_{3}$ contains three excitations and therefore it is not accessible to the propagation of a single photon. Counterrotating terms mix the one and three excitation sectors, opening the way to the appearance of this novel long-lived transmission resonance.

Notice that for $g \gtrsim 0.7$ a new regime seems to appear where the transmission is largely enhanced for a wide frequency range. This is reminiscent of the decoupling between light and matter predicted when $g \gtrsim 1$ in cavity QED [33]. However, the terms responsible for that decoupling, which involve only photon operators at the qubit position, are not present in our calculation, as they are expected to play a role only for larger $g$ 's than those considered here. The analysis of the transmission spectra at such high $g$ values, in the so-called "deep ultrastrong regime," is an interesting problem that is, however, beyond the aim of this work.

Inelastic scattering: Raman within just one photon.Figure 5 renders the transmitted flux at frequencies different to the incoming one, as a function of $\omega_{\text {in }}$. The Fourier analysis reveals that the frequency of the output flying photon is linked to $\omega_{\text {in }}$ through

$$
E_{G S}+\omega_{\text {in }}=E_{2}+\omega_{\text {out }} .
$$

Therefore, this inelastic process corresponds to a Raman scattering $[10,34]$ that leaves the dressed qubit in an excited bound state that, if counterrotating terms were not present, would fully reside in the sector $N_{\text {ext }}=2$. Within the RWA

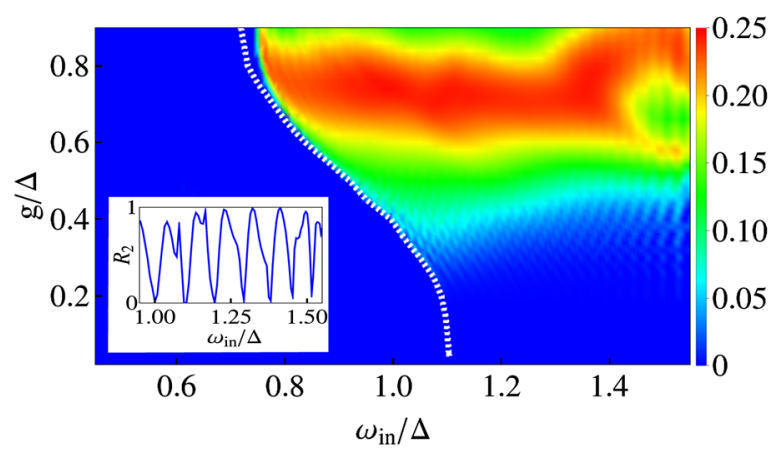

FIG. 5 (color online). Inelastic transmittance. Transmittance in the full model in the inelastic channel as a function of both incident photon frequency $\omega_{\text {in }}$ and $g$. The white line is the estimated boundary for the region where the photon frequency conversion occurs. The inset presents, for $g=0.8$, the inelastic reflection spectra when the waveguide is terminated at position $\Delta x=20$, showing that a $100 \%$ efficient Raman process is possible using one incoming photon. this sector is not accessible for one photon propagating in the GS, so this Raman process is genuine non-RWA physics.

As the output flying photon must belong to the onephoton band, the minimum frequency at which the Raman process may occur is $\min \left[\omega_{\text {Raman }}\right]=E_{2}-E_{G S}+1-2|J|$. The dependence with $g$ of this quantity is represented in Fig. 5 (white line), clearly marking the boundary for existence the inelastic transmission.

The computed inelastic transmittance never exceeds 0.25 . This turns out to be a fundamental upper bound: the maximization of the current in the inelastic channel, $P_{\text {ine }}$, subject to the conditions of current conservation $\left(1-|r|^{2}-|t|^{2}=P_{\text {ine }}\right)$, and continuity of the photonic wave function $(1+r=t)$, readily gives $\max \left[P_{\text {ine }}\right]=0.5$. As a pointlike qubit cannot differentiate between left and right, $P_{\text {ine }}$ is divided equally in both directions. This argument is analogous to that leading to the maximum possible absorption by pointlike scatterers [9]. Full absorption can be achieved in that case if a mirror is placed in the waveguide (the so-called "one-port coherent perfect absorption" [35]). Exploiting this analogy, we have considered the case where the waveguide is terminated at the transmission side of the qubit. In this case, "one-port coherent perfect Raman scattering," implying both photon frequency conversion and excitation of the dressed qubit, is possible with unit probability at the one-photon level, as shown in the inset of Fig. 5.

Both Fano and Raman processes described above are robust under moderate dissipation. Using a solvable model, that mimics dissipation by coupling the qubit to an auxiliary open waveguide, we have estimated that both processes should be visible at, at least, the dissipation levels present in some actual realizations in waveguide QED, such as superconducting circuits. Details on both the estimation and the model used can be found in the Supplemental Material.

It is interesting to analyze whether this Raman process may also occur in other systems. It is possible to show that it cannot occur if the qubit is substituted by a bosonic cavity or resonator, even if the coupling contains counterrotating terms (see Supplemental Material). This negative result can be traced back to the linearity of the Heisenberg equations for the bosonic creation operators. Therefore the system analyzed in this paper represents the minimal setup for observing inelastic scattering with a single photon.

Conclusions. - The scattering of a flying photon impinging into a two-level system placed in a waveguide has been studied for a large range of coupling strengths, including regimes were the rotating-wave approximation is no longer valid. For that, we have adapted the technique of matrix product states to scattering problems. Our results predict a rich phenomenology for the transmission spectra. At sufficiently small photon-qubit couplings the transmission spectra is dominated by a deep minimum, as found within 
the RWA. But when the coupling is strong enough, we predict new rich phenomenology for the transmission spectra: a blueshift in the transmission minima, appearance of a long-lived Fano resonance, and highly efficient inelastic processes. All these phenomena are due to the existence of bound multiphoton modes which are accessible when the full Hamiltonian is considered. The explored parameter range is accessible to the current experimental state of the art, at least using superconducting technology for both qubits and waveguides, thus opening the possibility to access nonperturbative quantum optics with single or few flying photons.

We acknowledge support by the Spanish Ministerio de Economia y Competitividad within projects MAT201128581-C02, FIS2012-33022, and No. FIS2011-25167, the Gobierno de Aragon (FENOL group), and the European project PROMISCE.

*1mm@unizar.es

[1] O. Astafiev, A. M. Zagoskin, A. A. Abdumalikov, Y. A. Pashkin, T. Yamamoto, K. Inomata, Y. Nakamura, and J. S. Tsai, Science 327, 840 (2010).

[2] I. C. Hoi, C. M. Wilson, G. Johansson, T. Palomaki, B. Peropadre, and P. Delsing, Phys. Rev. Lett. 107, 073601 (2011).

[3] M. F. Yanik, S. Fan, M. Soljačić, and J. D. Joannopoulos, Opt. Lett. 28, 2506 (2003).

[4] D. E. Chang, A.S. Sørensen, E. A. Demler, and M. D. Lukin, Nat. Phys. 3, 807 (2007).

[5] L. Zhou, H. Dong, Y. X. Liu, C. P. Sun, and F. Nori, Phys. Rev. A 78, 063827 (2008).

[6] L. Zhou, L.-P. Yang, Y. Li, and C. P. Sun, Phys. Rev. Lett. 111, 103604 (2013).

[7] E. Rephaeli and S. Fan, Phys. Rev. Lett. 108, 143602 (2012).

[8] D. Martín-Cano, A. González-Tudela, L. Martín-Moreno, F. J. García-Vidal, C. Tejedor, and E. Moreno, Phys. Rev. B 84, 235306 (2011).

[9] G. Romero, J. J. García-Ripoll, and E. Solano, Phys. Rev. Lett. 102, 173602 (2009).

[10] C. Cohen-Tannoudji, J. Dupont-Roc, and G. Grynberg, Atom-Photon Interactions: Basic Processes and Applications (Wiley-Interscience, New York, 1992) p. 680.

[11] C. Ciuti, G. Bastard, and I. Carusotto, Phys. Rev. B 72, 115303 (2005).
[12] K. Le Hur, Phys. Rev. B 85, 140506 (2012).

[13] G. Romero, D. Ballester, Y. M. Wang, V. Scarani, and E. Solano, Phys. Rev. Lett. 108, 120501 (2012).

[14] T. Niemczyk, F. Deppe, H. Huebl, E. Menzel, F. Hocke, M. Schwarz, J. J. García-Ripoll, D. Zueco, T. Hümmer, E. Solano, A. Marx, and R. Gross, Nat. Phys. 6, 772 (2010).

[15] J.-T. Shen and S. Fan, Phys. Rev. A 76, 062709 (2007).

[16] S. Fan, S. E. Kocabaş, and J. T. Shen, Phys. Rev. A 82, 063821 (2010).

[17] T. Shi, S. Fan, and C. P. Sun, Phys. Rev. A 84, 063803 (2011).

[18] H. Zheng and H. U. Baranger, Phys. Rev. Lett. 110, 113601 (2013).

[19] P. Longo, P. Schmitteckert, and K. Busch, J. Opt. A 11, 114009 (2009).

[20] P. Longo, P. Schmitteckert, and K. Busch, Phys. Rev. Lett. 104, 023602 (2010).

[21] G. Vidal, Phys. Rev. Lett. 91, 147902 (2003).

[22] G. Vidal, Phys. Rev. Lett. 93, 040502 (2004).

[23] F. Verstraete, J. J. García-Ripoll, and J. I. Cirac, Phys. Rev. Lett. 93, 207204 (2004).

[24] S. John and J. Wang, Phys. Rev. Lett. 64, 2418 (1990).

[25] J. J. García-Ripoll, New J. Phys. 8, 305 (2006).

[26] F. Verstraete, V. Murg, and J. I. Cirac, Adv. Phys. 57, 143 (2008).

[27] J. Eisert, M. Cramer, and M. B. Plenio, Rev. Mod. Phys. 82, 277 (2010).

[28] See Supplemental Material at http://link.aps.org/ supplemental/10.1103/PhysRevLett.113.263604 for (i) a brief overview on Matrix Product States, (ii) a discussion on the numerical convergence of our results and (iii) the analytical details on both the calculation of the frequency shift and the theorem on non-Raman scattering in linear systems, which includes Refs. [29,30].

[29] B. Pirvu, V. Murg, J. Cirac, and F. Verstraete, New J. Phys. 12, 025012 (2010).

[30] M. Suzuki, J. Math. Phys. (N.Y.) 32, 400 (1991).

[31] F. Guinea, E. Bascones, and M. J. Calderon, AIP Conf. Proc. 438, 1 (1998).

[32] B. Peropadre, D. Zueco, D. Porras, and J. J. García-Ripoll, Phys. Rev. Lett. 111, 243602 (2013).

[33] S. De Liberato, Phys. Rev. Lett. 112, 016401 (2014).

[34] R. W. Boyd, Nonlinear Optics, 2nd ed. (Academic Press, New York, 2003).

[35] Y. D. Chong, L. Ge, H. Cao, and A. D. Stone, Phys. Rev. Lett. 105, 053901 (2010). 